\title{
Product variety in Australian snacks and drinks: how can the consumer make a healthy choice?
}

\author{
Karen Z Walker ${ }^{1,2, *}$, Julie L Woods ${ }^{1}$, Cassie A Rickard ${ }^{1}$ and Carrie K Wong ${ }^{1}$ \\ ${ }^{1}$ Nutrition and Dietetics Unit, Department of Medicine, Monash University, Melbourne, Victoria, Australia: \\ ${ }^{2}$ Baker Heart Research Institute, PO Box 6492 St Kilda Road Central, Melbourne, Victoria 8008, Australia
}

Submitted 1 May 2007: Accepted 24 0ctober 2007: First published online 21 December 2007

\begin{abstract}
Objective: To estimate the proportion of 'healthy' snack food and beverage choices available to an Australian consumer.

Design: A survey of product Nutrition Information Panels (NIP) and product labels on snack foods and beverages offered for sale. Data on nutrient content were compared with criteria from different nutrient profile systems to estimate the proportion of items conforming to a 'healthy' choice.

Setting: A large supermarket in metropolitan Melbourne, Australia.

Results: A consumer could choose from 1070 different snack foods and 863 different drinks. Flavour variety was more common in snacks (maximum thirteen per product) while variation in container size was more common for drinks (up to ten per product). Recommended serving size for snacks varied greatly (18-100 g) while the serving size for drinks frequently did not correspond to the size of the container. Depending on the nutrient profile system selected, only 9-22\% of snack foods presented for sale could be deemed 'nutritious' by multiple criteria. Similarly, only $14-27 \%$ of beverages met 'healthy' criteria.

Conclusions: As one factor to help reduce the obesogenic environment, the supply balance needs to be shifted in favour of 'healthier' snack foods and beverages, e.g. by reformulation of many products by the food industry and their presentation in smaller, standardised portion-size packaging.
\end{abstract}

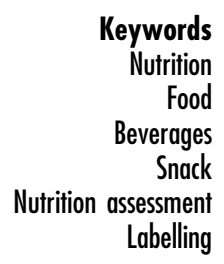

Obesity is a growing epidemic worldwide ${ }^{(1)}$. In Australia, half of men and nearly one-third of adult women are now overweight $^{(2)}$, as are about $20 \%$ of children and adolescents $^{(3)}$ and up to $15 \%$ of pre-school children ${ }^{(4)}$. The high prevalence of obesity brings considerable risk of chronic disease $^{(1)}$, imposes a considerable economic cost ${ }^{(5)}$ and presents a growing imperative to modify the predominantly obesogenic environment ${ }^{(6,7)}$. Adding to the effects of sedentary lifestyles ${ }^{(8)}$, many factors promote excessive food consumption ${ }^{(9)}$. These include aggressive food advertising, the ready availability and low price of energy-dense foods presented in large portion sizes, and the trend to consume more and more convenience foods away from home ${ }^{(10,11)}$. In Australia, expert opinion has identified the growing consumption of convenience foods as an important factor in the increase of obesity ${ }^{(7)}$. Moreover, convenience foods can erode an individual's control of his/her own diet, since it is the manufacturer who determines nutrient content and portion size ${ }^{(7)}$. Apart from take-away restaurant foods, snacks form one important category of convenience foods.

The term 'snack' is used in the present paper to refer to foods and drinks that can easily be consumed outside the context of the three main meals: breakfast, lunch and dinner ${ }^{(12,13)}$. Snack foods therefore are readily portable. Although nutrient content may be quite variable, snacks are often pleasurably savoury or sweet ${ }^{(14)}$ and some may be of high energy density ${ }^{(13)}$. Calorific beverages are often consumed with a food snack, providing considerable additional energy intake ${ }^{(15)}$. In contrast to meals, which are generally taken in the company of others, snacking can be a solitary activity ${ }^{(13)}$. Snack consumption in Australia is high ${ }^{(16)}$, as it is in the USA and other Western countries ${ }^{(17,18)}$, and may average nine snacks per week among adults ${ }^{(19)}$. Australian children also regularly consume snacks. A cross-sectional survey of school foods eaten by 5-12-year-old children in one region of Australia found that over $90 \%$ had energy-dense, micronutrientpoor snacks in their lunchbox ${ }^{(20)}$. Recommendations to reduce the intake of snack foods therefore form part of current weight management strategies directed at Australian children ${ }^{(21)}$. Australians also have a high consumption of soft drinks ${ }^{(22)}$ and in children this has been linked to obesity in later adolescence ${ }^{(23,24)}$.

The present study sought to quantify the availability, nutrient profile and cost of snack foods and beverages 
offered for sale in an Australian metropolitan setting, using data from the Nutrition Information Panel (NIP) displayed on all food and beverage packaging ${ }^{(25)}$. Consumers may not consult food labels ${ }^{(26)}$ or may have difficulty comparing the NIP on different foods to select healthy choices ${ }^{(27)}$. In addition, we argue here that the profusion of choice available makes finding 'healthy' snacks or drinks very difficult for the consumer. We have analysed this variety in order to determine the proportion of snacks and beverages that can be deemed 'healthy' using different nutrient profile models ${ }^{(28)}$, including the current New South Wales (NSW) Department of Health and UK 'traffic light' criteria ${ }^{(29,30)}$. We also provide data on variation in recommended serving size and nutrient content.

\section{Methods}

\section{Data collection}

In Australia, two supermarket chains currently control $80 \%$ of food sales. Surveys were undertaken to record information on the packaging of all snack foods and drinks presented for sale in a single large supermarket from one of these chains in metropolitan Melbourne. Data were collected as described previously ${ }^{(31)}$. A survey of all snacks (and fresh fruit) was conducted in September 2004 while information on beverages was collected in December 2006.

For this study, snacks were selected as non-perishable packaged foods that could be readily consumed outside a regular mealtime ${ }^{(12)}$. Foods that required utensils to eat (e.g. yoghurt) were excluded. Snacks were assessed in sixteen categories. Cakes \& pies included jam tarts, fruit pies, sponges and fruit cakes. Biscuits (cookies) were: (1) plain sweet - without fillings, icing or topping; (2) rich sweet - high in fat with nuts, dried fruit or chocolate chips and with fillings, topping or icing; or (3) chocolate chocolate-coated. Rich breads were muffins, iced buns, doughnuts, scones and croissants. Muesli bars were toasted grain bars ('health' or 'snack' bars). 'Breakfast bars' as replacement meals rather than snacks ${ }^{(31)}$ were excluded. Fruit slices and cereal slices had a cake base and a fruit/cereal filling. Chips $\&$ twisties included chips/ crisps and extruded twists or rings. Pretzels and popcorn included salted/sweetened varieties. Low-fat crackers and savoury biscuits contained $<5 \mathrm{~g}$ fat $/ 100 \mathrm{~g}$ and $>5 \mathrm{~g}$ fat/ $100 \mathrm{~g}$, respectively. Dips \& snacks were crackers packaged with a dip/spread. Fruit snacks were bars of dried fruit and/or sweetened fruit pulp. Dried fruit included banana chips and coated dried fruit clusters. Nut mixtures included seeds, coated nuts, and nut mixtures containing some dried fruit.

Beverages were grouped according to a proposed US beverage guidance system ${ }^{(15)}$. Seven types of drink were identified within Level 6 (calorically sweetened beverages), the least recommended category. The first of these, carbonated drinks, included 'soft drinks' (sugarsweetened beverages) as well as 'mixer drinks' or 'soft drinks' designed to be added to alcohol. Electrolyte drinks and formulated caffeinated drinks were drinks conforming to Standards $2 \cdot 6 \cdot 2$ and $2 \cdot 6 \cdot 4$, respectively, of the Australian and New Zealand Food Standards Code (ANZFSC) $^{(32)}$. Iced teas were drinks based on tea extract, flavoured with fruit juice. Cordials were sweet liquid or powdered concentrates to be made up with water. Fruit drinks (ANZFSC Standard $2 \cdot 6 \cdot 2^{(32)}$ ) contained $\geq 50 \mathrm{ml}$ fruit juice/1 while flavoured mineral waters were still or carbonated water flavoured with $<50 \mathrm{ml}$ fruit juice/l. Level 5 beverages were caloric beverages containing some nutrients ${ }^{(15)}$. Fruit juices could also contain a little vegetable juice and included carbonated varieties and non-alcoholic wines (grape juice). Vegetable juices were made solely from vegetables and included tomato juice. Full-cream milks came either from animal sources (ANZFSC Standard $2 \cdot 5 \cdot 1^{(32)}$ ) or from soy, rice or oats. Flavoured milks included all milk-based drinks with added sugars and flavours, while enriched milks were food supplements enriched with vitamins and minerals (ANZFSC Standard $2 \cdot 9 \cdot 3^{(32)}$ ). Powders for milk and powders for water were designed to be added to milk or water, respectively, the latter similar in taste and texture to a milk-based drink. Level 4 beverages were all non-caloric drinks ${ }^{(15)}$, including all artificially sweetened carbonated beverages and liquid or powdered cordial concentrates as well as a few diet varieties of formulated caffeinated drinks, electrolyte drinks or fruit drinks. Level 3 beverages consisted of low-fat milks ${ }^{(15)}$ from animal sources (ANZFSC Standard $2 \cdot 5 \cdot 1^{(32)}$ ) or other reducedfat milks of plant origin. Level 2 beverages (tea and coffee) ${ }^{(15)}$ were excluded. Level 1 beverages ${ }^{(15)}$ were plain waters in still or carbonated form.

Data taken from the product label and the NIP were recorded on standardised entry sheets as described elsewhere $^{(31)}$. Nutrient content for powdered or concentrated products was based on the final drink made up according to the manufacturer's instructions. Data for powders for milk have been based on drinks made up with reducedfat cow's milk. Where the same beverage was present in many bottle sizes, nutrient and cost information were taken from the bottle at or nearest in size to one litre.

\section{Data analysis}

Data were analysed using the Statistical Package for the Social Sciences (SPSS for Windows) statistical software package version $14 \cdot 0 \cdot 1$ (SPSS Inc., Chicago, IL, USA). Items in each food/beverage category were assessed as the sum of products and product varieties (flavour alternatives for a given product). For beverages, bottles or packs of different sizes were also counted. Due to nonsymmetrical distribution of data, aggregates are presented as the median and interquartile range. 
Table 1 Quantity, variety and serving size of snack foods and drinks available for sale at a Melbourne supermarket. Serving size for fresh fruit is also given. Total number represents the number of products plus product varieties

\begin{tabular}{|c|c|c|c|c|c|c|c|c|}
\hline Snacks & $\begin{array}{c}\text { Total } \\
\text { number }\end{array}$ & $\begin{array}{c}\text { Percentage } \\
\text { with varieties* }\end{array}$ & $\begin{array}{l}\text { Serving } \\
\text { size }(g) \dagger\end{array}$ & $\begin{array}{l}\text { Beverages } \\
\text { (level) } \ddagger\end{array}$ & $\begin{array}{c}\text { Total } \\
\text { number }\end{array}$ & $\begin{array}{c}\text { Percentage } \\
\text { with varieties* }\end{array}$ & $\begin{array}{l}\text { Percentage in } \\
\text { multiple sizes* }\end{array}$ & $\begin{array}{c}\text { Serving } \\
\text { size }(m \mathrm{l}) \dagger\end{array}$ \\
\hline Cakes \& pies & 83 & $23(4)$ & $44(15)$ & Calorically sweetened (6) & & & & \\
\hline Sweet biscuits & & & & Carbonated/flavoured & 106 & $13(5)$ & $31(10)$ & $250(0)$ \\
\hline Plain sweet & 69 & $17(13)$ & $18(23)$ & Electrolyte & 26 & $27(8)$ & $19(3)$ & $250(50)$ \\
\hline Rich sweet & 155 & $19(7)$ & $23(23)$ & Formulated caffeinated & 9 & $55(3)$ & 77 (4) & $250(73)$ \\
\hline Chocolate & 22 & $14(13)$ & $35(6)$ & Iced teas & 11 & $64(4)$ & $36(3)$ & $275(95)$ \\
\hline Rich breads & 29 & $24(11)$ & $100(70)$ & Cordials & 69 & $29(10)$ & $33(3)$ & $250(50)$ \\
\hline Muesli bars & 93 & $27(8)$ & $34(14)$ & Fruit drinks & 73 & $22(6)$ & $26(4)$ & $250(50)$ \\
\hline Fruit slices & 55 & $29(6)$ & $38(5)$ & Flavoured mineral waters & 20 & $30(5)$ & $15(2)$ & $250(38)$ \\
\hline Cereal slices & 28 & $25(6)$ & $45(41)$ & Caloric with nutrients (5) & & & & \\
\hline Chips \& twisties & 147 & $22(9)$ & $50(5)$ & Fruit juices & 217 & $20(8)$ & $33(5)$ & $200(50)$ \\
\hline Pretzels & 9 & $22(3)$ & $50(0)$ & Vegetable juices & 9 & $11(3)$ & $33(3)$ & $225(50)$ \\
\hline Popcorn & 15 & $40(3)$ & $80(70)$ & Full-fat plain milks & 22 & $16(2)$ & $41(6)$ & $250(0)$ \\
\hline Low-fat crackers & 90 & $21(10)$ & $25(18)$ & Flavoured milks & 42 & $26(6)$ & $24(7)$ & $250(0)$ \\
\hline Savoury biscuits & 120 & $49(10)$ & $35(16)$ & Enriched milks & 13 & $31(5)$ & 31 (3) & $250(0)$ \\
\hline Dips \& snacks & 25 & $28(4)$ & $26(17)$ & Powders for milk & 36 & $25(5)$ & $28(5)$ & 219 (16) \\
\hline Fruit snacks & 69 & $26(6)$ & $20(10)$ & Powders for water & 63 & $29(8)$ & $8(2)$ & $188(29)$ \\
\hline Dried fruit & 20 & $10(3)$ & $40(30)$ & Non-caloric (4) & & & & \\
\hline Nut mixtures & 41 & $10(3)$ & $40(15)$ & Diet beverages $\S$ & 75 & $19(7)$ & $35(10)$ & $250(550)$ \\
\hline \multirow[t]{3}{*}{ Fresh fruit } & & & $142(68)$ & Milks (3) & & & & \\
\hline & & & & $\begin{array}{l}\text { Reduced-fat } \\
\text { Waters (1) }\end{array}$ & 32 & $0(1)$ & $37(5)$ & $250(0)$ \\
\hline & & & & Still/carbonated & 40 & $3(3)$ & $50(8)$ & $250(0)$ \\
\hline
\end{tabular}

*Maximum number per product given in parentheses.

tMedian values are given with the interquartile range in parentheses.

$\ddagger$ Based on the US beverage guidance system ${ }^{(15)}$.

§Includes low-calorie cordials ( $n$ 26), carbonated drinks ( $n$ 41), formulated caffeinated drinks $(n 3)$, fruit drinks $(n 3)$, one iced tea, one electrolyte drink.

To determine the proportion of snacks that could be designated as 'healthy', snacks were assessed according to criteria developed by the NSW Department of Health and Department of Education and Training for school canteens ${ }^{(29)}$; and by criteria developed for an Australian food company ${ }^{(19)}$. In addition, snacks were also assessed by the UK signpost criteria ${ }^{(30)}$ defining the green/amber (low/medium) or amber/red (medium/high) boundaries. Beverages were assessed by the NSW canteen criteria ${ }^{(29)}$ (energy $\leq 300 \mathrm{~kJ} /$ serving, sodium $\leq 100 \mathrm{mg} /$ serving). As data were taken only from beverage packs of approximately one litre capacity, these criteria were applied to the recommended serving sizing rather than to the packet as sold. Beverages were also assessed by the UK signpost criteria ${ }^{(30)}$.

\section{Results}

One thousand and seventy different snacks were available for selection in this one supermarket location (Table 1). Cakes \& pies, sweet biscuits and rich breads made up the greatest proportion of these snack items (Fig. 1). Overall $25 \%$ of snacks came in multiple flavour varieties (maximum of thirteen varieties per product) (Table 1). Flavour variety was greatest for savoury biscuits (49\% with varieties) and least evident for dried fruit or nut mixtures (only $10 \%$ had varieties). In all snacks, there was a marked lack of uniformity in recommended serving sizes. These ranged from a median of $18 \mathrm{~g}$ (for plain sweet

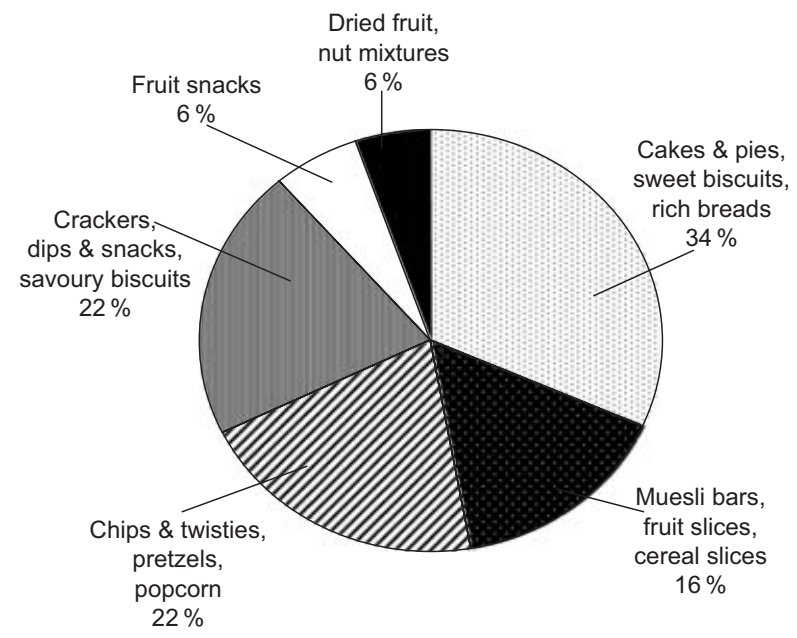

Fig. 1 Proportion of snack food items in different categories found at a metropolitan supermarket in Australia

biscuits) to $100 \mathrm{~g}$ (sweet breads). A median serving of fresh fruit (142 g) was appreciably larger than any serving recommended for snack foods.

A consumer also had the choice of 863 different drinks (Table 1), only $14 \%$ of which were non-calorific (Level 1 or 4 ) and only $4 \%$ of which were nutritious (Level 3) (Fig. 2). Beverages came in up to ten flavour varieties, with the most variety evident in the enriched milks and flavoured waters. Drink variety was also greatly expanded by the presence of multiple bottle or pack sizes. Overall, $28 \%$ of drinks came in more than one size, with up to ten 
different sizes present for a given item. Despite the variety of bottle or pack size, the recommended serving size given for most drinks was $250 \mathrm{ml}$. The smallest median serving size was for powdered drinks designed to be made up with water $(188 \mathrm{ml})$ while the largest was for iced tea $(275 \mathrm{ml})$.

For snack foods, the highest median energy content per serving was found in popcorn, rich breads and chips \& twisties (Table 2). Chips \& twisties also had the highest median content per serving of total and saturated fat. Pretzels and dips \& snacks had the highest median content of sodium. For beverages, the flavoured milks and enriched milks had the highest median energy

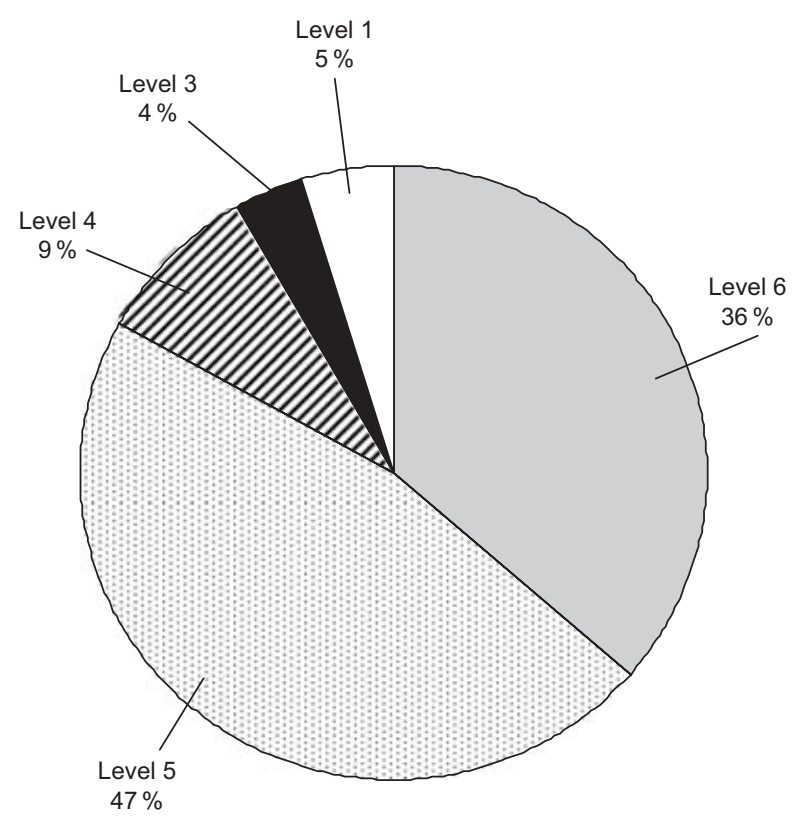

Fig. 2 Proportion of beverages in different nutritional categories $^{(15)}$ found at a metropolitan supermarket in Australia content per serving (Table 3). Enriched milks also had the highest median content per serving of protein and total carbohydrate. The formulated caffeinated drinks had the highest median content of sugars and sodium.

Snack foods were assessed to determine how many were compliant with criteria developed by the NSW State Government for school canteens ${ }^{(29)}$ (Table 4). Many snacks complied with at least one criterion, but overall only $22 \%$ were compliant with all three criteria for a 'nutritious' snack (i.e. low energy, low saturated fat and low sodium). Fruit snacks showed the highest percentage overall compliance $(67 \%)$. When a criterion for dietary fibre content $(>0.3 \mathrm{~g} / 100 \mathrm{~kJ})$ was applied to snacks where information was available on the NIP (only 540 of 1070 items), the proportion of snacks deemed 'nutritious' by all four criteria fell to only 9\%. Application of a different nutrient profile developed for a food manufacturer ${ }^{(19)}$ (energy $<600 \mathrm{~kJ} /$ serving, saturated fat $<2 \mathrm{~g} /$ serving, sodium $<300 \mathrm{mg} / 100 \mathrm{~g}$ ) gave similar results to the analysis in relation to NSW canteen criteria, in that overall only $20 \%$ of snack foods were compliant for all three criteria for a 'nutritious' snack (data not shown).

Both the NSW school canteen criteria and the manufacturer's criteria assess foods on a nutrient per serving basis. Yet there are very large variations in serving size between snacks that may affect results (Table 1). Snacks were therefore also assessed by the UK signpost criteria $^{(30)}$ comparing nutrients in a constant weight of food (Table 4). Overall, by this assessment only $1 \%$ of snacks met all four 'green' criteria (fat $\leq 3 \mathrm{~g} / 100 \mathrm{~g}$, saturated fat $\leq 1.5 \mathrm{~g} / 100 \mathrm{~g}$, total sugars $\leq 5 \mathrm{~g} / 100 \mathrm{~g}$ and salt $\leq 0.3 \mathrm{~g} /$ $100 \mathrm{~g}$ ) while only 9\% avoided qualifying for any 'red' signs. Some foods that met all three NSW canteen criteria still attracted 'red' labels by UK signpost criteria; e.g. biscuits, rich breads, muesli bars and fruit slices. Conversely, more pretzels, popcorn, low-fat crackers

Table 2 The macronutrient content of snacks found in an Australian supermarket. Values are given as median (interquartile range)

\begin{tabular}{|c|c|c|c|c|c|c|}
\hline Snacks & $\begin{array}{c}\text { Energy } \\
\text { (kJ/serving) }\end{array}$ & $\begin{array}{c}\text { Protein } \\
\text { (g/serving) }\end{array}$ & $\begin{array}{c}\text { Total fat } \\
\text { (g/serving) }\end{array}$ & $\begin{array}{c}\text { Saturated fat } \\
\text { (g/serving) }\end{array}$ & $\begin{array}{c}\text { Carbohydrate } \\
\text { (g/serving) }\end{array}$ & $\begin{array}{l}\text { Sodium } \\
(\mathrm{mg} / 100 \mathrm{~g})\end{array}$ \\
\hline Cakes \& pies & $657(282)$ & $2 \cdot 0(1 \cdot 1)$ & $6 \cdot 2(4 \cdot 5)$ & $2 \cdot 3(1 \cdot 8)$ & $24 \cdot 1(10 \cdot 8)$ & 350 (157) \\
\hline \multicolumn{7}{|l|}{ Sweet biscuits } \\
\hline Plain sweet & $336(413)$ & $1 \cdot 2(1 \cdot 4)$ & $3.2(3.5)$ & $1 \cdot 5(1 \cdot 3)$ & $13 \cdot 2(14 \cdot 5)$ & $283(226)$ \\
\hline Rich sweet & $452(461)$ & $1 \cdot 2(1 \cdot 2)$ & $4 \cdot 4(5 \cdot 2)$ & $2 \cdot 3(2 \cdot 7)$ & $14.1(14.9)$ & 264 (172) \\
\hline Chocolate & 731 (123) & $2 \cdot 1(0.6)$ & $8.5(3.9)$ & $4 \cdot 2(1 \cdot 6)$ & $21.0(6.0)$ & $210(205)$ \\
\hline Rich breads & $1452(1147)$ & $5 \cdot 0(4 \cdot 6)$ & $10 \cdot 2(14.9)$ & $3 \cdot 0(5 \cdot 1)$ & $49 \cdot 3(31 \cdot 2)$ & $338(111)$ \\
\hline Muesli bars & 637 (285) & $2.4(1.9)$ & $5 \cdot 1(6 \cdot 1)$ & $2 \cdot 1(2 \cdot 5)$ & $21 \cdot 5(4 \cdot 4)$ & 100 (155) \\
\hline Fruit slices & $547(86)$ & $1.8(0.5)$ & $1 \cdot 4(2 \cdot 3)$ & $0.5(1.2)$ & $26.4(3.4)$ & $158(89)$ \\
\hline Cereal slices & 702 (997) & $2 \cdot 1(1 \cdot 8)$ & $4 \cdot 4(16 \cdot 4)$ & $1 \cdot 2(7 \cdot 0)$ & $29 \cdot 4(17 \cdot 0)$ & $183(211)$ \\
\hline Chips \& twisties & 1044 (117) & $4.4(0.9)$ & $14.2(4.0)$ & $6 \cdot 4(5 \cdot 6)$ & $25 \cdot 6(6 \cdot 3)$ & $652(443)$ \\
\hline Pretzels & 811 (101) & $5.0(2.0)$ & $1.6(2.9)$ & $0.3(0.4)$ & $38.0(3.0)$ & $1200(590)$ \\
\hline Popcorn & 1635 (1328) & $4 \cdot 3(6 \cdot 3)$ & $9 \cdot 4(20 \cdot 0)$ & $3.2(7 \cdot 6)$ & $35.6(30.9)$ & 780 (967) \\
\hline Low-fat crackers & 397 (315) & $2.0(1.7)$ & $0.5(0.6)$ & $0.1(0)$ & $19 \cdot 8(15 \cdot 8)$ & 395 (412) \\
\hline Savoury biscuits & $576(361)$ & $3.0(1.8)$ & $3.9(3.4)$ & $1 \cdot 1(2 \cdot 2)$ & $18.9(11.4)$ & 778 (468) \\
\hline Dips \& snacks & $495(314)$ & $3.0(2.7)$ & $6 \cdot 8(5 \cdot 1)$ & $3.5(2.7)$ & $9 \cdot 2(7 \cdot 0)$ & $906(441)$ \\
\hline Fruit snacks & 314 (239) & $0.4(0.7)$ & $0.8(0.8)$ & $0.5(0.7)$ & $15 \cdot 6(4 \cdot 3)$ & 110 (127) \\
\hline Dried fruit & 487 (441) & $0.8(1.1)$ & $0.3(5.4)$ & $0.1(5.0)$ & $24 \cdot 2(17 \cdot 4)$ & 37 (81) \\
\hline Nut mixtures & $820(450)$ & $5.0(3.0)$ & $13.9(11 \cdot 6)$ & $1 \cdot 5(2 \cdot 3)$ & $3.6(15.6)$ & $11(55)$ \\
\hline TOTAL & 596 (435) & $2 \cdot 1(2 \cdot 2)$ & $4.7(7 \cdot 8)$ & $1.7(3.1)$ & $21.0(13.7)$ & $320(423)$ \\
\hline
\end{tabular}


Table 3 The macronutrient content of beverages offered for sale in an Australian supermarket. Values are given as median (interquartile range)

\begin{tabular}{|c|c|c|c|c|c|c|}
\hline Beverages (level)* & $\begin{array}{c}\text { Energy } \\
\text { (kJ/serving) }\end{array}$ & $\begin{array}{l}\text { Protein } \\
\text { (g/serving) }\end{array}$ & $\begin{array}{c}\text { Carbohydrate } \\
\text { (g/serving) }\end{array}$ & $\begin{array}{l}\text { Total sugars } \\
\text { (g/serving) }\end{array}$ & $\begin{array}{l}\text { Total fat } \\
\text { (g/serving) }\end{array}$ & $\begin{array}{l}\text { Sodium } \\
(\mathrm{mg} / 100 \mathrm{~g})\end{array}$ \\
\hline \multicolumn{7}{|l|}{ Calorically sweetened (6) } \\
\hline Carbonated/flavoured & $394(127)$ & $0(0)$ & $23 \cdot 0(7 \cdot 1)$ & $22 \cdot 8(8 \cdot 0)$ & $0(0)$ & $18(10)$ \\
\hline Electrolyte & $270(13)$ & $0(0)$ & $15 \cdot 0(0 \cdot 2)$ & $15 \cdot 0(0 \cdot 8)$ & $0(0)$ & $58(62)$ \\
\hline Formulated caffeinated & $480(106)$ & $0 \cdot 1(0 \cdot 6)$ & $28 \cdot 3(5 \cdot 8)$ & $28 \cdot 0(3 \cdot 8)$ & $0 \cdot 1(0 \cdot 6)$ & 165 (237) \\
\hline Iced teas & 465 (183) & $0.1(0.1)$ & $27 \cdot 3(16 \cdot 2)$ & $27 \cdot 3(10 \cdot 7)$ & $0.0(0.1)$ & $17(12)$ \\
\hline Cordials & $350(52)$ & $0 \cdot 0(1 \cdot 0)$ & $20.5(3.5)$ & $20 \cdot 1(3 \cdot 4)$ & $0.0(1.0)$ & $10(5)$ \\
\hline Fruit drinks & 467 (164) & $1 \cdot 0(1 \cdot 1)$ & $27 \cdot 6(9 \cdot 9)$ & $27 \cdot 5(9 \cdot 1)$ & $0.2(1.0)$ & $13(17)$ \\
\hline $\begin{array}{l}\text { Flavoured mineral } \\
\text { waters }\end{array}$ & $113(150)$ & $0 \cdot 0(0 \cdot 8)$ & $6 \cdot 3(7 \cdot 3)$ & $6 \cdot 3(7 \cdot 4)$ & $0.0(0 \cdot 8)$ & $10(8)$ \\
\hline \multicolumn{7}{|l|}{ Caloric with nutrients (5) } \\
\hline Fruit juices & $396(90)$ & $1 \cdot 0(0 \cdot 0)$ & $22 \cdot 0(6 \cdot 1)$ & $21 \cdot 0(5 \cdot 9)$ & $1 \cdot 0(1 \cdot 0)$ & $12(9)$ \\
\hline Vegetable juices & $190(44)$ & $2 \cdot 0(1.5)$ & $0.25(0.8)$ & $8.7(2.9)$ & $0.3(0.8)$ & 350 (269) \\
\hline Full-fat plain milks & $664(26)$ & $8 \cdot 0(0.3)$ & $12 \cdot 0(1 \cdot 0)$ & $12 \cdot 0(1 \cdot 0)$ & $8.5(0.9)$ & $118(45)$ \\
\hline Flavoured milks & 705 (323) & $8.0(1 \cdot 8)$ & $22 \cdot 0(9 \cdot 3)$ & $22 \cdot 0(10 \cdot 8)$ & $4.5(3.9)$ & $133(60)$ \\
\hline Enriched milks & $850(277)$ & $12 \cdot 5(11 \cdot 0)$ & $31 \cdot 0(14 \cdot 5)$ & $18.5(5 \cdot 9)$ & $3 \cdot 8(1 \cdot 4)$ & $188(118)$ \\
\hline Powders for milk & 642 (182) & $7 \cdot 9(1 \cdot 6)$ & $23 \cdot 0(6 \cdot 8)$ & $19 \cdot 7(4 \cdot 9)$ & $3 \cdot 3(0 \cdot 8)$ & $127(28)$ \\
\hline Powders for water & 252 (132) & $1 \cdot 8(1 \cdot 4)$ & $10 \cdot 0(4 \cdot 1)$ & $7 \cdot 8(5 \cdot 8)$ & $1 \cdot 6(1 \cdot 8)$ & $69(82)$ \\
\hline \multicolumn{7}{|l|}{ Non-caloric (4) } \\
\hline Diet beverages & $18(24)$ & $0.0(0.1)$ & $0 \cdot 8(1 \cdot 3)$ & $0.5(1.8)$ & $0(0)$ & $20(15)$ \\
\hline \multicolumn{7}{|l|}{ Milks (3) } \\
\hline Reduced-fat & 459 (152) & $8.5(1.5)$ & $13 \cdot 0(2 \cdot 1)$ & $13 \cdot 0(2 \cdot 1)$ & $2 \cdot 2(3 \cdot 0)$ & $129(42)$ \\
\hline \multicolumn{7}{|l|}{ Waters (1) } \\
\hline Still/carbonated & $0(0)$ & $0(0)$ & $0(0)$ & $0(0)$ & $0(0)$ & $0(6)$ \\
\hline
\end{tabular}

*Based on the US beverage guidance system ${ }^{(15)}$.

Table 4 Percentage of snack foods complying with New South Wales (NSW) canteen criteria ${ }^{(29)}$ or with the green/amber boundary in UK nutritional signpost labelling ${ }^{(30)}$

\begin{tabular}{|c|c|c|c|c|c|c|c|c|c|}
\hline \multirow[b]{3}{*}{ Category } & \multicolumn{9}{|c|}{ Percentage of foods in each category compliant with criteria } \\
\hline & \multicolumn{4}{|c|}{ NSW canteen criteria } & \multicolumn{5}{|c|}{ Criteria on the UK nutritional signpost labelling } \\
\hline & $\begin{array}{c}\text { Energy } \\
<600 \mathrm{~kJ} / \text { serving } \\
(<900 \mathrm{~kJ} / \text { serving })\end{array}$ & $\begin{array}{l}\text { Saturated fat } \\
<3 \mathrm{~g} / \text { serving }\end{array}$ & $\begin{array}{c}\text { Sodium } \\
<200 \mathrm{mg} / 100 \mathrm{~g}\end{array}$ & $\begin{array}{c}\text { All } 3 \\
\text { criteria }\end{array}$ & $\begin{array}{l}\text { Total fat } \\
\text { Green: } \\
\leq 3 \mathrm{~g} / 100 \mathrm{~g}\end{array}$ & $\begin{array}{l}\text { Saturated fat } \\
\text { Green: } \\
\leq 1.5 \mathrm{~g} / 100 \mathrm{~g}\end{array}$ & $\begin{array}{c}\text { Sugars } \\
\text { Green: } \\
\leq 5 \mathrm{~g} / 100 \mathrm{~g}\end{array}$ & $\begin{array}{c}\text { Salt } \\
\text { Green: } \\
\leq 0.3 \mathrm{~g} / 100 \mathrm{~g}\end{array}$ & $\begin{array}{c}\text { All greent } \\
\text { (no red) } \ddagger\end{array}$ \\
\hline Cakes \& pies* & 84 & 59 & 16 & 5 & 6 & 10 & 1 & 41 & $1(1)$ \\
\hline \multicolumn{10}{|l|}{ Biscuits } \\
\hline Plain sweet & 59 & 83 & 33 & 20 & 3 & 4 & 0 & 19 & $0(0)$ \\
\hline Rich sweet & 38 & 61 & 27 & 17 & 0 & 6 & 0 & 11 & $0(0)$ \\
\hline Chocolate & 27 & 81 & 48 & 10 & 0 & 0 & 0 & 18 & $0(0)$ \\
\hline Rich breads* & 31 & 48 & 7 & 3 & 7 & 13 & 3 & 0 & $0(0)$ \\
\hline Muesli bars & 43 & 66 & 74 & 23 & 4 & 7 & 0 & 56 & $0(1)$ \\
\hline Fruit slices* & 91 & 71 & 67 & 46 & 48 & 50 & 0 & 15 & $0(0)$ \\
\hline Cereal slices* & 68 & 68 & 61 & 36 & 21 & 32 & 0 & 18 & $0(0)$ \\
\hline $\begin{array}{l}\text { Chips \& } \\
\quad \text { twisties }\end{array}$ & 15 & 30 & 9 & 0 & 1 & 1 & 91 & 7 & $1(1)$ \\
\hline Pretzels & 0 & 100 & 0 & 0 & 9 & 89 & 89 & 0 & $0(11)$ \\
\hline Popcorn & 0 & 47 & 13 & 0 & 15 & 13 & 80 & 7 & $0(13)$ \\
\hline $\begin{array}{l}\text { Low-fat } \\
\text { crackers }\end{array}$ & 89 & 100 & 17 & 17 & 69 & 90 & 88 & 7 & $6(67)$ \\
\hline $\begin{array}{l}\text { Savoury } \\
\text { biscuits }\end{array}$ & 51 & 81 & 1 & 0 & 0 & 27 & 74 & 0 & $0(18)$ \\
\hline Dips \& snacks & 64 & 40 & 8 & 4 & 4 & 4 & 64 & 4 & $0(4)$ \\
\hline Fruit snacks & 90 & 91 & 77 & 67 & 22 & 41 & 1 & 57 & $0(1)$ \\
\hline Dried fruit & 55 & 65 & 95 & 40 & 65 & 60 & 0 & 80 & $0(0)$ \\
\hline Nut mixtures & 22 & 71 & 85 & 22 & 48 & 10 & 54 & 80 & $0(2)$ \\
\hline TOTAL & 53 & 67 & 33 & 22 & 14 & 22 & 34 & 20 & $1(9)$ \\
\hline
\end{tabular}

${ }^{\star}$ Energy criterion used for this group was $<900 \mathrm{~kJ} /$ serving.

tAll green: complying with the criteria given in columns to the left.

$\ddagger$ No red: compliant with four out of four criteria - total fat $\leq 20 \mathrm{~g} / 100 \mathrm{~g}$, saturated fat $\leq 5 \mathrm{~g} / 100 \mathrm{~g}$, sugars $\leq 15 \mathrm{~g} / 100 \mathrm{~g}$ and salt $<1.5 \mathrm{~g} / 100 \mathrm{~g}$.

and savoury biscuits satisfied UK signpost criteria than multiple NSW canteen criteria.

When beverages were assessed by NSW canteen criteria (energy $\leq 300 \mathrm{~kJ} /$ serving, sodium $\leq 100 \mathrm{mg} /$ serving),
$27 \%$ met both criteria. Our analysis, however, was based on recommended serving size whereas this nutrient profile was really designed to apply to the whole carton or bottle as sold in canteens, and not applicable in our 
Table 5 Proportion of beverages in different categories complying with the nutritional criteria applying to drinks in the UK signposting recommendations ${ }^{(30)}$

\begin{tabular}{|c|c|c|c|c|c|c|c|c|c|}
\hline \multirow[b]{3}{*}{ Category } & \multicolumn{9}{|c|}{ Percentage compliant with recommendation } \\
\hline & \multicolumn{2}{|c|}{$\begin{array}{l}\text { Total fat } \\
\text { per } 100 \mathrm{~g}\end{array}$} & \multicolumn{2}{|c|}{$\begin{array}{l}\text { Saturated fat } \\
\text { per } 100 \mathrm{~g}\end{array}$} & \multicolumn{2}{|c|}{$\begin{array}{l}\text { Sugars } \\
\text { per } 100 \mathrm{~g}\end{array}$} & \multicolumn{2}{|c|}{$\begin{array}{l}\text { Sodium } \\
\text { per } 100 \mathrm{~g}\end{array}$} & \multirow[b]{2}{*}{$\begin{array}{l}\text { Meeting all } \\
\text { criteria for green }\end{array}$} \\
\hline & $\begin{array}{l}\text { Green: } \\
<1.5 \mathrm{~g}\end{array}$ & $\begin{array}{l}\text { Amber: } \\
1 \cdot 5-10 \mathrm{~g}\end{array}$ & $\begin{array}{l}\text { Green: } \\
<0.75 \mathrm{~g}\end{array}$ & $\begin{array}{c}\text { Amber: } \\
<0.75-2.5 \mathrm{~g}\end{array}$ & $\begin{array}{l}\text { Green: } \\
<2.5 \mathrm{~g}\end{array}$ & $\begin{array}{l}\text { Amber: } \\
2 \cdot 5-7 \cdot 5 \mathrm{~g}\end{array}$ & $\begin{array}{l}\text { Green: } \\
<118 \mathrm{mg}\end{array}$ & $\begin{array}{c}\text { Amber: } \\
118-590 \mathrm{mg}\end{array}$ & \\
\hline \multicolumn{10}{|l|}{ Calorically sweetened (6) } \\
\hline Carbonated/flavoured & 100 & 0 & 100 & 0 & 0 & 13 & 100 & 0 & 0 \\
\hline Electrolyte & 100 & 0 & 100 & 0 & 0 & 69 & 100 & 0 & 0 \\
\hline Formulated caffeinated & 100 & 0 & 78 & 22 & 0 & 0 & 0 & 100 & 0 \\
\hline Iced teas & 100 & 0 & 100 & 0 & 0 & 55 & 100 & 0 & 0 \\
\hline Cordials & 100 & 0 & 84 & 16 & 0 & 10 & 100 & 0 & 0 \\
\hline Fruit drinks & 100 & 0 & 100 & 0 & 0 & 0 & 100 & 0 & 0 \\
\hline $\begin{array}{l}\text { Flavoured mineral } \\
\text { waters }\end{array}$ & 100 & 0 & 100 & 0 & 20 & 80 & 100 & 0 & 20 \\
\hline \multicolumn{10}{|l|}{ Caloric with nutrients (5) } \\
\hline Fruit juices & 100 & 0 & 96 & 4 & 0 & 4 & 100 & 0 & 0 \\
\hline Vegetable juices & 56 & 44 & 100 & 0 & 33 & 67 & 11 & 89 & 11 \\
\hline Full-fat plain milks & 0 & 100 & 0 & 77 & 0 & 100 & 100 & 0 & 0 \\
\hline Flavoured milks & 17 & 83 & 33 & 67 & 0 & 29 & 0 & 0 & 0 \\
\hline Enriched milks & 15 & 85 & 69 & 31 & 0 & 85 & 100 & 0 & 0 \\
\hline Powders for milk & 28 & 72 & 0 & 100 & 0 & 19 & 97 & 3 & 0 \\
\hline Powders for water & 71 & 29 & 49 & 51 & 29 & 64 & 100 & 0 & 24 \\
\hline \multicolumn{10}{|l|}{ Non-caloric (4) } \\
\hline Diet beverages & 100 & 0 & 81 & 19 & 93 & 7 & 99 & 1 & 75 \\
\hline \multicolumn{10}{|l|}{ Milks (3) } \\
\hline Reduced-fat & 84 & 16 & 50 & 50 & 0 & 100 & 28 & 72 & 0 \\
\hline \multicolumn{10}{|l|}{ Waters (1) } \\
\hline Still/carbonated & 100 & 0 & 100 & 0 & 100 & 0 & 100 & 0 & 100 \\
\hline TOTAL & 86 & 14 & 80 & 20 & 16 & 24 & 90 & 5 & 14 \\
\hline
\end{tabular}

study. Beverages therefore were also assessed by the more detailed UK signpost criteria $^{(30)}$ that are applied on a per $100 \mathrm{~g}$ basis (Table 5). In this analysis, overall, only $1 \%$ of Level 6 beverages and $4 \%$ of Level 5 beverages met all four 'low' criteria.

\section{Discussion}

The present study indicates that an Australian consumer, entering a large metropolitan supermarket to select a snack or beverage, is overwhelmed by food choice (Table 1). While our study examines only one store, there is considerable uniformity between stores in Australia due to the dominance of only two supermarket chains. Nutrient content (Tables 2 and 3) clearly shows that the product diversity is generally not nutritionally beneficial (capable of enhancing overall diet quality) ${ }^{(33)}$ but is mere product diversity aimed at increasing sales ${ }^{(34)}$. 'Line extensions' (new flavours for a well-established product), 'me-too' foods (mirroring rival products) and multiple packaging are strategies employed to stimulate consumer demand $^{(35)}$ even though this is driving an obesity epidemic $^{(18)}$. Among overwhelming product diversity how is the consumer to select 'healthy' snacks and drinks?

'Nutrient profiling' or the categorising of foods in terms of nutritional composition ${ }^{(28)}$ can aid consumers ${ }^{(36)}$ although there is debate about the optimal system ${ }^{(37)}$. In the USA, where food labels report the content of many micronutrients ${ }^{(38)}$, continuous nutrient indices such as the Naturally Nutrient Rich (NNR) score may be used ${ }^{(39)}$. In Australia, this approach is precluded since only macronutrient and sodium content are mandatory ${ }^{(25)}$. If the NIP carried information on calcium, zinc, iron, magnesium and folate, micronutrients of importance in snacks ${ }^{(19)}$, this would aid consumer choice and allow a continuous nutrient index to be developed for local use.

The 'healthiness' of snack foods and beverages was first assessed against macronutrient-related criteria recently developed in New South Wales as a tool for planning school canteen menus ${ }^{(29)}$. This nutrient profile was consistent with four of five inclusion criteria used in other studies in that it is published, has clear guidelines, includes use of data on more than one nutrient and is based on absolute nutrient values ${ }^{(37)}$. One limitation, however, is that this profile was developed for schoolchildren rather than for adults. Only $22 \%$ of snacks and $27 \%$ of beverages available on the supermarket shelf fulfilled the multiple criteria of this nutrient profile. Another limitation in applying the NSW canteen guidelines is that these criteria depend on highly variable serving size (Table 1 ) potentially open to manipulation by manufacturers to improve the appearance of the nutrient profile. The consumer may also consume the whole packet or drink an entire beverage carton or bottle of drink rather than limit their consumption to a single designated serving ${ }^{(38)}$. If serving-based nutrient profiling is employed, there is a strong need for serving sizes to be standardised.

UK signpost criteria $^{(39)}$ based on nutrient content per $100 \mathrm{~g}$ were also applied to snacks (Table 4), markedly reducing the number of categories high in 'healthy' items. It was also of interest that while many fruit snacks passed 
the three NSW canteen criteria, fruit snacks by UK signpost criteria usually bore a 'red' high sugar warning. This indicates the importance of the sugar criterion, since fruit snacks include foods such as 'roll-ups', energy-dense snacks of fruit paste, sugars and maltodextrin, that are arguably not healthy. To avoid excluding whole fruit, however, any sugar criterion applied generally to foods should specify added sugar(39) or non-milk extrinsic sugar $^{(37)}$.

The US guidance system for beverages ${ }^{(15)}$ proved useful for profiling Australian beverages and served to indicate how caloric Level 5 and 6 beverages dominate supermarket shelves (Table 1, Fig. 2). These drinks are not satiating and may promote excessive energy intake and weight gain ${ }^{(15)}$. The NSW canteen nutrient profiles ${ }^{(29)}$ proved less applicable to supermarket beverages than to snacks owing to their reliance on container size as sold. UK signpost recommendations ${ }^{(30)}$ avoid this difficulty and when applied indicate that only $14 \%$ of beverages are 'healthy' by multiple criteria (Table 5).

There have been recent calls for the adoption of UK 'traffic light' signals on Australian foods ${ }^{(39)}$ and the present data (Tables 4 and 5) support the utility of this system. However, while clear front-of-package labelling is important to guide consumer choice, other measures must also be considered to reduce the overwhelming preponderance of nutrient-poor snacks and beverages on supermarket shelves. The Australian food industry can be innovative and there have been initiatives made to reformulate snacks ${ }^{(40)}$. A strong response is now urgently needed to create a diversity of snacks and beverages with reduced energy density, and reduced amounts of fat, salt and added sugar ${ }^{(18)}$, presented to consumers in standardised portion size packs ${ }^{(11)}$.

\section{Acknowledgements}

The authors have no conflict of interest to declare. C.A.R. was in receipt of a Monash University Vacation Scholarship, otherwise the study received no external funding. All authors collected the data with the help of BND students at Monash University. C.A.R., C.K.W. and K.Z.W. were responsible for data analysis. The first draft of the manuscript was prepared by K.Z.W. and J.L.W., while C.A.R. and C.K.W. reviewed the literature and helped revise the manuscript before submission. We thank Emma Boschetti, Jamie Ross and other students in BND2052 for their contribution towards data collection.

\section{References}

1. Isganaitis E \& Lustig R (2005) Fast food, central nervous system insulin resistance and obesity. Arterioscler Thromb Vasc Biol 25, 2451-2462.

2. Thorburn A (2005) Prevalence of obesity in Australia. Obes $\operatorname{Rev}$ 6, 187-189.
3. Bauer L (2002) Child and adolescent obesity in the 21st century: an Australian perspective. Asia Pac J Clin Nutr 11, Suppl. 3, S524-S528.

4. Wake M, Hardy P, Canterford L, Sawyer M \& Carlin JB (2007) Overweight, obesity and girth of Australian preschoolers: prevalence and socio-economic correlates. Int J Obes (Lond) 31, 1044-1051.

5. Yates J \& Murphy C (2006) A cost benefit analysis of weight management strategies. Asia Pac J Clin Nutr 15, Suppl., 74-79.

6. Bray G \& Champagne C (2005) Beyond energy balance: there is more to obesity than kilocalories. J Am Diet Assoc 105, Suppl. 1, S17-S23.

7. Banwell C, Hinde S, Dixon J \& Sibthorpe B (2005) Reflections on expert consensus: a case study on the social trends contributing to obesity. Eur J Public Health 15, 564-568.

8. Egger G, Swinburn B \& Rossner S (2003) Dusting off the epidemiological triad: could it work with obesity? Obes Rev 4, 115-119.

9. Silventoinen K, Sans S, Tolonen H, Monterde D, Kuulasmaa K, Kesteloot H \& Tuomilehto J (2004) Trends in obesity and energy supply in the WHO MONICA project. Int $J$ Obes Relat Metab Dis 28, 710-718.

10. Drewnowski A \& Specter SE (2004) Poverty and obesity: the role of energy density and energy costs. Am J Clin Nutr 79, 6-16.

11. French S, Story M \& Jeffrey R (2001) Environmental influences on eating and physical activity. Annu Rev Public Health 22, 309-335.

12. Drummond S, Crombie N \& Kirk T (1996) A critique of the effects of snacking on body weight status. Eur J Clin Nutr 50, 779-783.

13. de Graaf C (2006) Effects of snacks on energy intake: an evolutionary perspective. Appetite 47, 18-23.

14. Chamontin A, Pretzer G \& Booth D (2003) Ambiguity of 'snack' in British usage. Appetite 41, 21-29.

15. Popkin BM, Armstrong LE, Bray GM, Caballero B, Frei B \& Willett WC (2006) A new proposed guidance system for beverage consumption in the United States. Am J Clin Nutr 83, 529-542.

16. McLennan W \& Podger A (1997) National Nutrition Survey 1995: Selected Highlights. Canberra: Australian Bureau of Statistics.

17. Johns L, Siega-Riz A \& Popkin B (2001) The increasing prevalence of snacking among US children from 1977 to 1996. J Pediatr 138, 493-498.

18. Astrup A, Bovy M, Nacenhorst K \& Popova A (2006) Food for thought or thought for food? A stakeholder dialogue around the role of the snacking industry in addressing the obesity epidemic. Obes Rev 7, 303-312.

19. Tapsell L, Williams P, Droulez V, Gillen L \& Murray S (2006) A Nutritious Snacks Initiative for Uncle Toby's. Wollongong: National Centre of Excellence in Functional Foods.

20. Sanigorski AM, Bell AC, Kremer PJ \& Swinburn BA (2005) Lunchbox contents of Australian school children: room for improvement. Eur J Clin Nutr 59, 1310-1316.

21. Gehling RK, Magarey AM \& Daniels LA (2005) Food-based recommendations to reduce fat intake: an evidence-based approach to the development of a family-focused child weight management programme. J Paediatr Child Health 41, 112-118.

22. Gill TP, Rangan AM \& Webb KL (2006) The weight of evidence suggests that soft drinks are a major issue in childhood and adolescent obesity. There is much to be gained by reducing children's intake of soft drinks and littleexcept excess weight - to be lost. Med J Aust 184, 263-264.

23. Tam CS, Garnett SP, Cowell CT, Campbell K, Cabrera G \& Baur LA (2006) Soft drink consumption and excess weight 
gain in Australian school students: results from the Nepean study. Int J Obes (Lond) 30, 1091-1093.

24. St Onge M-P, Keller K \& Heymsfield S (2003) Changes in childhood food consumption patterns: a cause for concern in light of increasing body weights. Am J Clin Nutr $\mathbf{7 8}$, 1068-1073.

25. Curran M (2002) Nutrition labelling: perspectives of a bi-national agency for Australia and New Zealand. Asia Pac J Clin Nutr 11, S72-S76.

26. Cowburn G \& Stockley L (2005) Consumer understanding and use of nutrition labelling: a systematic review. Public Health Nutr 8, 21-28.

27. Baines J \& Lata S (2004) Consumer understanding and use of nutrition information panels. Asia Pac J Clin Nutr 13, Suppl., S160.

28. Scarborough P, Rayner M \& Stockley L (2007) Developing nutrient profile models: a systematic approach. Public Health Nutr 10, 330-336.

29. Andrews R, Mathews R, Burt S \& Stanton R (2006) Fresh tastes@ school. NSW Healthy Canteen Strategy. Canteen Menu Planning Guide, 2nd ed. Sydney: NSW Department of Health and NSW Department of Education and Training.

30. UK Food Standards Agency (2007) Front of Pack Nutritional Signpost Labelling. Technical Guidance. http:// www.food.gov.uk/multimedia/pdfs/frontofpackguidance. pdf (accessed May 2007).

31. Woods J \& Walker KZ (2007) Choosing breakfast: how well does packet information on Australian breakfast cereals, bars and drinks reflect recommendations? Nutr Diet 64, 226.

32. Food Standards Australia New Zealand (2006) Australia New Zealand Food Standards Code. Canberra: FSANZ.

33. Thiele S, Mensink G \& Bleitz R (2004) Determinants of diet quality. Public Health Nutr 7, 29-37.

34. Costa A \& Jongen WMF (2006) New insights into consumerled food product development. Trends Food Sci Technol 17, 457-465.

35. Linnemann A, Benner M, Verkerk R \& van Boekel M (2006) Consumer-driven food product development. Trends Food Sci Technol 17, 184-190.

36. Kant A (2000) Consumption of energy dense, nutrient-poor foods by adult Americans: nutritional and health implications. The First National Health and Nutrition Examination Survey, 1998-1994. Am J Clin Nutr 72, 929-936.

37. Scarborough P, Boxer A, Rayner M \& Stockley L (2007) Testing nutrient profile models using data from a survey of nutrition professionals. Public Health Nutr 10, 337-345.

38. Pelletier A, Chang W, Delzell J \& McCall J (2004) Patients' understanding and use of snack food nutrition labels. $J \mathrm{Am}$ Board Fam Pract 17, 319-323.

39. Beard T, Nowson C \& Riley M (2007) Traffic-light food labels. Med J Aust 186, 19.

40. National Food Industry Strategy (2007) FIG case study Manildra Group. http://www.nfis.com.au/FIG_case_study (accessed July 2007). 